\title{
Water-Level Altitudes in Wells Completed in the Northern Segment of the Edwards Aquifer, Travis, Williamson, and Bell Counties, Texas, March-June 2005
}

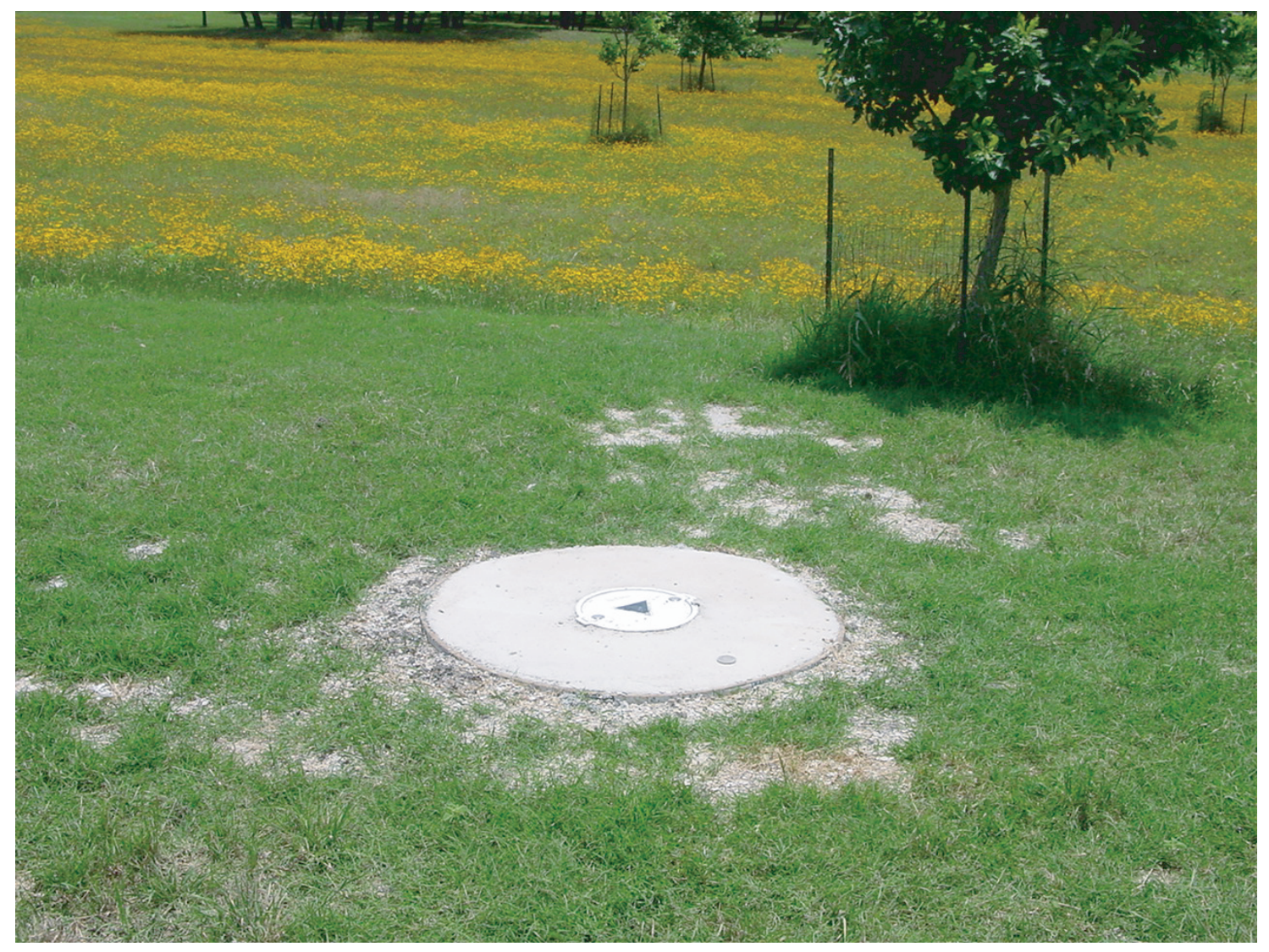

Data Series 125 
Cover. Edwards aquifer well in southern Williamson County (photograph by M.L. Greenslate, U.S. Geological Survey, June 2005). 


\section{Water-Level Altitudes in Wells Completed in the Northern Segment of the Edwards Aquifer, Travis, Williamson, and Bell Counties, Texas, March-June 2005}

By Sachin D. Shah

In cooperation with the Texas Commission on Environmental Quality

Data Series 125 


\section{U.S. Department of the Interior Gale A. Norton, Secretary}

\section{U.S. Geological Survey \\ P. Patrick Leahy, Acting Director}

\section{U.S. Geological Survey, Reston, Virginia: 2005}

For sale by U.S. Geological Survey, Information Services

Box 25286, Denver Federal Center

Denver, CO 80225

For more information about the USGS and its products:

Telephone: 1-888-ASK-USGS

World Wide Web: http://www.usgs.gov/

Any use of trade, product, or firm names in this publication is for descriptive purposes only and does not imply endorsement by the U.S. Government.

Although this report is in the public domain, permission must be secured from the individual copyright owners to reproduce any copyrighted materials contained within this report. 


\section{Contents}

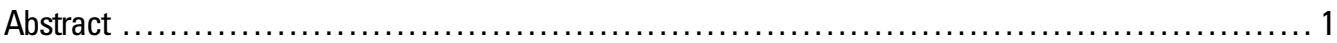

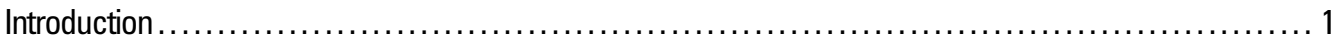

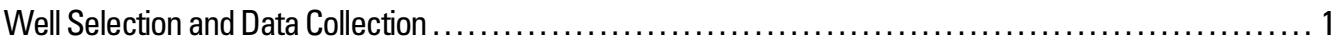

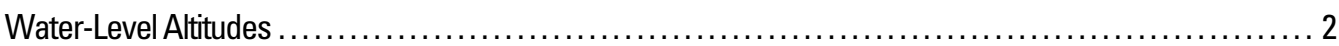

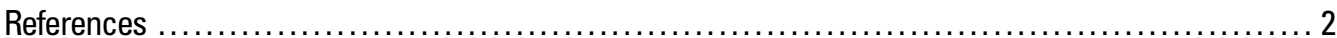

\section{Figure}

1. Map showing northern segment of the Edwards aquifer and sites of wells in which water levels

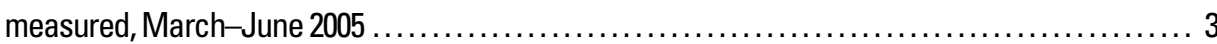

\section{Table}

1. Well locations and water-level altitudes in the northern segment of the Edwards aquifer, March-June 2005

\section{Vertical and Horizontal Datums}

Vertical coordinate information is referenced to the North American Vertical Datum of 1988 (NAVD 88).

Horizontal coordinate information is referenced to the North American Datum of 1983 (NAD 83). 


\title{
Water-Level Altitudes in Wells Completed in the Northern Segment of the Edwards Aquifer, Travis, Williamson, and Bell Counties, Texas, March-June 2005
}

\author{
By Sachin D. Shah
}

\section{Abstract}

During March-June 2005, the U.S. Geological Survey, in cooperation with the Texas Commission on Environmental Quality (TCEQ), measured water levels in 80 Edwards aquifer wells in the outcrop of the northern segment of the aquifer in Travis, Williamson, and Bell Counties. Public-supply and private wells were selected in areas where outcrop water-level data were sparse. The altitude data can be used in the TCEQ Source Water Assessment and Protection program to help delineate zones of capture around sources of public water supply. Altitudes range from about 490 to about 935 feet above North American Vertical Datum of 1988.

\section{Introduction}

This report, prepared by the U.S. Geological Survey (USGS) in cooperation with the Texas Commission on Environmental Quality (TCEQ), presents water-level altitudes obtained from 80 wells completed in the outcrop of the northern segment of the Edwards aquifer in Travis, Williamson, and Bell Counties (fig. 1) during March-June 2005. The objective of the study was to compile a database of water-level altitudes for the TCEQ Source Water Assessment and Protection (SWAP) program in parts of the outcrop area where water-level-altitude data are sparse. The TCEQ implemented the SWAP program to determine the susceptibility to chemical constituents of the State's public water sources (Texas Natural Resource Conservation Commission, 2005). The altitude data can be used to help delineate zones of capture around sources.

The Edwards aquifer in Central Texas commonly is divided into the San Antonio segment, the Barton Springs segment, and the northern segment (fig. 1). The northern segment consists of the Edwards aquifer north of the Colorado River. Baker and others (1986) presents a comprehensive description of the geohydrology of the northern segment of the Edwards aquifer. From that report, the outcrop of the northern segment encompasses an area of about 500 square miles and consists mostly of limestone with some interbedded marl. These rocks typically are dense, grayish to white, and massive. Where present, soils on the outcrop typically are brown, silty to clayey loams that range in thickness from negligible to about 5 feet. The thickness of the northern segment of the aquifer, which has been influenced by erosion and faulting, ranges from negligible to about 450 feet. From the outcrop, the aquifer dips to the eastsoutheast at an average slope of 70 to 75 feet per mile beneath rocks that confine the Edwards aquifer and yield little to no water. Recharge to the northern Edwards aquifer occurs primarily by downward percolation of rainfall on the outcrop. Solution features such as honeycombing, sinkholes, and caverns allow for rapid infiltration of water. Water levels fluctuate over a wide range in most areas because of the rapid rates of recharge and, to a lesser extent, variations in rates of discharge.

The author acknowledges well owners for allowing USGS personnel access to their property and their wells.

\section{Well Selection and Data Collection}

Wells for water-level measurement were selected from available databases of well information compiled by the Texas Water Development Board (2005), the TCEQ (J. Meyer, Texas Commission on Environmental Quality, written commun., 2005), and the USGS (M.L. Greenslate, U.S. Geological Survey, written commun., 2005). Public-supply and private wells were selected in areas where outcrop water-level data were sparse. On the basis of available well depth and aquifer top and bottom altitude data, only wells completed in the Edwards aquifer outcrop were included. If well depth was unavailable, the decision to include a well was made on the basis of other information in the database. The wells selected for water-level measurement have comparable depths and screened intervals. Access to wells was provided by municipalities, private owners, 


\section{Water-Level Altitudes in Wells Completed in the Northern Segment of the Edwards Aquifer, Texas, March-June 2005}

and ground-water conservation districts. Water-level measurements were obtained by electronic sensor, steel tape, or calibrated airline. Public-supply wells are pumped daily but were shut down for 12 hours prior to measurement so that static water-level measurements could be made.

\section{Water-Level Altitudes}

Water-level altitudes and associated data for the 80 wells in which measurements were made during March-June 2005 are listed in table 1. Altitudes range from about 490 to about 935 feet above NAVD 88. Depths to water range from about 5 to 265 feet below land surface. Well depths among wells with known depths range from 26 to 450 feet below land surface.

\section{References}

Ashworth, J.B, and Hopkins, Janie, 1995, Aquifers of Texas: Texas Water Development Board Report 345, 69 p.

Baker, E.T., Slade, R.M., Jr., Dorsey, M.E., Ruiz, L.M., and Duffin, G.L., 1986, Geohydrology of the Edwards aquifer in the Austin area, Texas: Texas Water Development Board Report 293, 215 p.

Texas Natural Resource Conservation Commission [former name of TCEQ], 2005, Source Water Assessment and Protection program (SWAP)—SWAP objectives and overview: accessed July 1, 2005, at http://www.tnrcc.state.tx.us/ permitting/waterperm/pdw/swap/swap.html

Texas Water Development Board, 2005, Water information, integration, \& dissemination: accessed July 1, 2005, at http://www.twdb.state.tx.us/home/index.asp 


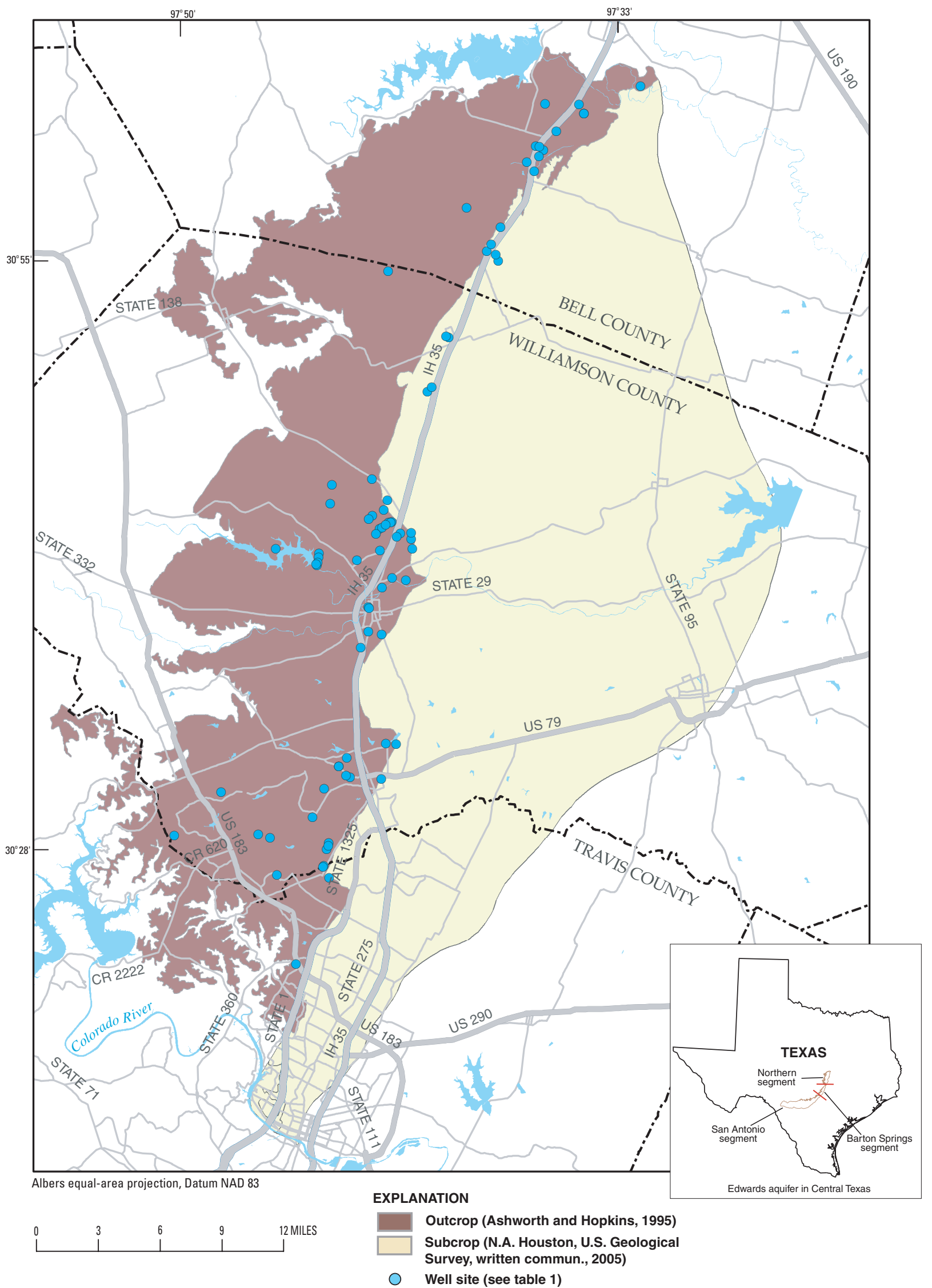

Figure 1. Map showing northern segment of the Edwards aquifer and sites of wells in which water levels measured, March-June 2005. 
Table 1. Well locations and water-level altitudes in the northern segment of the Edwards aquifer, March-June 2005.

[dd, degrees; mm, minutes; ss, seconds; --, no data]

\begin{tabular}{|c|c|c|c|c|c|c|c|c|c|c|}
\hline $\begin{array}{c}\text { State } \\
\text { well } \\
\text { number }\end{array}$ & $\begin{array}{l}\text { USGS site } \\
\text { identifier }\end{array}$ & $\begin{array}{c}\text { Latitude } \\
\text { (ddmmss.ss) }\end{array}$ & $\begin{array}{c}\text { Longitude } \\
\text { (ddmmss.ss) }\end{array}$ & $\begin{array}{l}\text { Well depth } \\
\text { (feet below } \\
\text { land } \\
\text { surface) }\end{array}$ & $\begin{array}{l}\text { Water } \\
\text { use }\end{array}$ & $\begin{array}{c}\text { Land-surface } \\
\text { altitude } \\
\text { (feet above } \\
\text { NAVD 88) }\end{array}$ & $\begin{array}{l}\text { Drill } \\
\text { date }\end{array}$ & $\begin{array}{c}\text { Water-level } \\
\text { measurement } \\
\text { date }\end{array}$ & $\begin{array}{c}\text { Water level } \\
\text { (feet below } \\
\text { land } \\
\text { surface) }\end{array}$ & $\begin{array}{c}\text { Water-level } \\
\text { altitude } \\
\text { (feet above } \\
\text { NAVD 88) }\end{array}$ \\
\hline \multicolumn{11}{|c|}{ Travis County } \\
\hline $58-35-403$ & 302720097431701 & 302720.00 & 974317.00 & 95.0 & domestic & 840.0 & 1964 & $04 / 16 / 2005$ & 62.76 & 777.19 \\
\hline $58-35-405$ & 302717097431901 & 302717.00 & 974319.00 & 100.0 & unused & 845.0 & 1959 & $04 / 26 / 2005$ & 57.93 & 784.71 \\
\hline $58-35-415$ & 302652097430501 & 302649.00 & 974303.00 & 112.0 & stock & 830.0 & -- & $04 / 26 / 2005$ & 90.28 & 739.89 \\
\hline $58-35-702$ & 302257097444701 & 302312.00 & 974444.00 & 49.0 & unused & 873.0 & 1935 & $05 / 12 / 2005$ & 22.96 & 846.58 \\
\hline \multicolumn{11}{|c|}{ Williamson County } \\
\hline $58-18-605$ & 304046097452001 & 304045.00 & 974519.00 & 140.0 & domestic & 856.60 & 2005 & 06/06/2005 & 14.16 & 842.44 \\
\hline 58-19-104 & 304324097423001 & 304324.00 & 974230.00 & 140.0 & domestic & 801.31 & 2005 & 06/06/2005 & 48.12 & 753.19 \\
\hline $58-19-105$ & 304247097431101 & 304236.00 & 974235.00 & 100.0 & domestic & 794.80 & 2005 & $04 / 26 / 2005$ & 73.93 & 720.87 \\
\hline 58-19-204 & 304336097403201 & 304336.00 & 974032.00 & 126.0 & domestic & 810.0 & 05-20-1976 & $05 / 11 / 2005$ & 61.06 & 729.69 \\
\hline $58-19-316$ & 304242097394801 & 304242.00 & 973948.00 & -- & public supply & 739.17 & -- & $04 / 26 / 2005$ & 49.56 & 689.61 \\
\hline $58-19-407$ & 304031097431201 & 304031.00 & 974312.00 & 86.3 & domestic & 870.06 & -- & 05/13/2005 & 62.95 & 807.11 \\
\hline $58-19-408$ & 304021097431301 & 304021.00 & 974313.00 & 86.3 & domestic & 852.44 & -- & $05 / 13 / 2005$ & 51.60 & 800.84 \\
\hline $58-19-409$ & 304001097431901 & 304001.00 & 974319.00 & 45.0 & domestic & 781.02 & -- & 05/13/2005 & 11.30 & 769.72 \\
\hline $58-19-410$ & 304008097431601 & 304008.00 & 974316.00 & 26.0 & domestic & 802.85 & -- & $05 / 13 / 2005$ & 49.60 & 753.25 \\
\hline $58-19-411$ & 304003097432101 & 304003.00 & 974321.00 & 45.0 & domestic & 782.65 & -- & 05/13/2005 & 11.90 & 770.75 \\
\hline $58-19-502$ & 304203097403201 & 304203.00 & 974032.00 & 124.0 & domestic & 740.0 & 1975 & $04 / 26 / 2005$ & 71.60 & 667.95 \\
\hline 58-19-506 & 304154097404401 & 304154.00 & 974044.00 & 110.0 & domestic & 750.0 & 1978 & $05 / 11 / 2005$ & 54.74 & 700.15 \\
\hline $58-19-507$ & 304118097403101 & 304117.00 & 974023.00 & 185.0 & public supply & 770.0 & 05-11-1979 & $04 / 26 / 2005$ & 36.50 & 722.34 \\
\hline $58-19-518$ & 304130097401201 & 304130.00 & 974012.00 & -- & domestic & 742.72 & -- & 05/13/2005 & 62.99 & 679.73 \\
\hline $58-19-519$ & 304132097400601 & 304132.00 & 974006.00 & 115.0 & domestic & 733.63 & -- & 05/13/2005 & 56.44 & 677.19 \\
\hline $58-19-521$ & 304036097401301 & 304036.00 & 974013.00 & -- & public supply & 728.59 & -- & $04 / 26 / 2005$ & 73.46 & 655.13 \\
\hline $58-19-522$ & 304012097412101 & 304011.00 & 974121.00 & 130.0 & domestic & 751.60 & 2005 & $06 / 06 / 2005$ & 51.81 & 699.79 \\
\hline $58-19-603$ & 304139097395501 & 304139.00 & 973955.00 & 72.0 & irrigation & 700.0 & -- & $04 / 26 / 2005$ & 54.29 & 651.72 \\
\hline 58-19-619 & 304117097384001 & 304117.00 & 973840.00 & 220.0 & public supply & 691.0 & 03-18-1985 & 05/10/2005 & 22.00 & 670.24 \\
\hline $58-19-626$ & 304217097400001 & 304217.00 & 974000.00 & 105.0 & irrigation & 705.0 & 04-06-1984 & 05/10/2005 & 21.52 & 689.27 \\
\hline $58-19-628$ & 304108097392401 & 304108.00 & 973924.00 & 200.0 & public supply & 733.0 & $12-05-1986$ & $05 / 10 / 2005$ & 38.69 & 695.69 \\
\hline
\end{tabular}


Table 1. Well locations and water-level altitudes in the northern segment of the Edwards aquifer, March-June 2005-Continued.

\begin{tabular}{|c|c|c|c|c|c|c|c|c|c|c|}
\hline $\begin{array}{c}\text { State } \\
\text { well } \\
\text { number }\end{array}$ & $\begin{array}{l}\text { USGS site } \\
\text { identifier }\end{array}$ & $\begin{array}{c}\text { Latitude } \\
\text { (ddmmss.ss) }\end{array}$ & $\begin{array}{l}\text { Longitude } \\
\text { (ddmmss.ss) }\end{array}$ & $\begin{array}{c}\text { Well depth } \\
\text { (feet below } \\
\quad \text { land } \\
\text { surface) }\end{array}$ & $\begin{array}{l}\text { Water } \\
\text { use }\end{array}$ & $\begin{array}{c}\text { Land-surface } \\
\text { altitude } \\
\text { (feet above } \\
\text { NAVD 88) }\end{array}$ & $\begin{array}{l}\text { Drill } \\
\text { date }\end{array}$ & $\begin{array}{l}\text { Water-level } \\
\text { measurement } \\
\text { date }\end{array}$ & $\begin{array}{c}\text { Water level } \\
\text { (feet below } \\
\text { land } \\
\text { surface) }\end{array}$ & $\begin{array}{c}\text { Water-level } \\
\text { altitude } \\
\text { (feet above } \\
\text { NAVD 88) }\end{array}$ \\
\hline 58-19-632 & 304102097384001 & 304102.00 & 973840.00 & -- & stock & 660.0 & -- & $05 / 10 / 2005$ & 22.00 & 640.69 \\
\hline $58-19-633$ & 304117097391201 & 304117.00 & 973912.00 & -- & domestic & 679.80 & -- & $05 / 13 / 2005$ & 4.82 & 674.98 \\
\hline $58-19-634$ & 304145097394601 & 304145.00 & 973946.00 & -- & irrigation & 688.10 & -- & $05 / 13 / 2005$ & 3.93 & 684.17 \\
\hline $58-19-635$ & 304146097393701 & 304146.00 & 973937.00 & -- & irrigation & 693.85 & -- & $05 / 13 / 2005$ & 13.90 & 679.95 \\
\hline $58-19-636$ & 304145097394001 & 304145.00 & 973940.00 & -- & irrigation & 688.82 & -- & $05 / 13 / 2005$ & 5.26 & 683.56 \\
\hline $58-19-637$ & 304037097383801 & 304037.00 & 973838.00 & -- & domestic & 712.16 & -- & $04 / 26 / 2005$ & 39.01 & 673.15 \\
\hline $58-19-802$ & 303812097405001 & 303812.00 & 974050.00 & 102.0 & unused & 750.0 & 1912 & $04 / 26 / 2005$ & 75.25 & 666.45 \\
\hline $58-19-803$ & 303809097404901 & 303809.00 & 974049.00 & 186.0 & public supply & 750.0 & 1952 & $04 / 26 / 2005$ & 73.92 & 670.31 \\
\hline $58-19-805$ & 303859097400901 & 303901.00 & 974009.00 & 175.0 & public supply & 680.0 & 12-09-1957 & $04 / 26 / 2005$ & 55.44 & 611.91 \\
\hline 58-19-901 & 303919097385801 & 303919.00 & 973858.00 & 184.0 & irrigation & 680.0 & 1971 & $05 / 10 / 2005$ & 35.93 & 645.77 \\
\hline 58-19-914 & 303925097393801 & 303925.00 & 973938.00 & 100.0 & domestic & 705.0 & -- & $05 / 10 / 2005$ & 50.98 & 649.86 \\
\hline $58-26-808$ & 303032097481501 & 303032.00 & 974814.00 & 150.0 & domestic & 874.91 & 2005 & $06 / 06 / 2005$ & 60.78 & 814.13 \\
\hline $58-27-213$ & 303702097401301 & 303702.00 & 974013.00 & 205.0 & domestic & 775.0 & 04-06-1973 & $04 / 25 / 2005$ & 113.19 & 645.37 \\
\hline $58-27-214$ & 303629097411601 & 303629.00 & 974116.00 & 100.0 & public supply & 785.0 & 03-08-1977 & $05 / 11 / 2005$ & 88.88 & 693.97 \\
\hline $58-27-221$ & 303710097405101 & 303710.00 & 974051.00 & 200.0 & public supply & 753.0 & 1982 & $04 / 26 / 2005$ & 69.30 & 680.65 \\
\hline $58-27-536$ & 303247097403201 & 303247.00 & 974032.00 & 140.0 & domestic & 716.49 & 2005 & $06 / 29 / 2005$ & 59.55 & 656.94 \\
\hline $58-27-610$ & 303230097395901 & 303230.00 & 973959.00 & 140.0 & domestic & 717.97 & 2005 & $06 / 29 / 2005$ & 53.84 & 664.13 \\
\hline $58-27-721$ & 303104097431601 & 303036.00 & 974311.00 & 120.0 & domestic & 797.81 & 2005 & $04 / 22 / 2005$ & 48.00 & 749.81 \\
\hline $58-27-814$ & 303101097415401 & 303101.00 & 974154.00 & 222.0 & public supply & 750.0 & 1940 & $04 / 22 / 2005$ & 27.70 & 722.28 \\
\hline $58-27-819$ & 303117097421301 & 303108.00 & 974206.00 & 203.0 & public supply & 749.0 & 07-25-1978 & $04 / 22 / 2005$ & 37.43 & 711.96 \\
\hline $58-27-824$ & 303132097422601 & 303132.00 & 974226.00 & 135.0 & public supply & 775.0 & 05-11-1979 & $04 / 22 / 2005$ & 46.62 & 719.69 \\
\hline $58-27-829$ & 303132097422602 & 303132.00 & 974226.00 & 140.0 & public supply & 775.0 & 03-15-1979 & $04 / 22 / 2005$ & 47.52 & 719.69 \\
\hline $58-27-838$ & 303152097420301 & 303152.00 & 974203.00 & 115.0 & domestic & 745.74 & 2005 & $06 / 06 / 2005$ & 27.90 & 717.84 \\
\hline $58-27-839$ & 303057097402301 & 303057.00 & 974023.00 & 190.0 & unused & 702.65 & -- & $04 / 14 / 2005$ & 20.04 & 682.61 \\
\hline 58-34-119 & 302844097503501 & 302844.00 & 975034.00 & 60.0 & domestic & 976.43 & 2005 & $06 / 06 / 2005$ & 40.75 & 935.68 \\
\hline $58-34-305$ & 302834097455101 & 302833.00 & 974552.00 & 65.0 & unused & 895.0 & 04-19-1980 & $04 / 22 / 2005$ & 14.41 & 871.04 \\
\hline 58-34-309 & 302925097462001 & 302925.00 & 974620.00 & 140.0 & domestic & 910.10 & 2005 & $06 / 29 / 2005$ & 46.10 & 864.00 \\
\hline $58-34-621$ & 302707097461101 & 302700.00 & 974536.00 & 180.0 & domestic & 889.88 & 2005 & $04 / 22 / 2005$ & 14.57 & 875.31 \\
\hline
\end{tabular}


Table 1. Well locations and water-level altitudes in the northern segment of the Edwards aquifer, March-June 2005-Continued.

\begin{tabular}{|c|c|c|c|c|c|c|c|c|c|c|}
\hline $\begin{array}{c}\text { State } \\
\text { well } \\
\text { number }\end{array}$ & $\begin{array}{l}\text { USGS site } \\
\text { identifier }\end{array}$ & $\begin{array}{c}\text { Latitude } \\
\text { (ddmmss.ss) }\end{array}$ & $\begin{array}{c}\text { Longitude } \\
\text { (ddmmss.ss) }\end{array}$ & $\begin{array}{l}\text { Well depth } \\
\text { (feet below } \\
\text { land } \\
\text { surface) }\end{array}$ & $\begin{array}{l}\text { Water } \\
\text { use }\end{array}$ & $\begin{array}{c}\text { Land-surface } \\
\text { altitude } \\
\text { (feet above } \\
\text { NAVD 88) }\end{array}$ & $\begin{array}{l}\text { Drill } \\
\text { date }\end{array}$ & $\begin{array}{l}\text { Water-level } \\
\text { measurement } \\
\text { date }\end{array}$ & $\begin{array}{l}\text { Water level } \\
\text { (feet below } \\
\text { land } \\
\text { surface) }\end{array}$ & $\begin{array}{c}\text { Water-level } \\
\text { altitude } \\
\text { (feet above } \\
\text { NAVD 88) }\end{array}$ \\
\hline $58-35-102$ & 302817097430101 & 302818.00 & 974301.00 & 46.0 & industrial & 775.0 & -- & $04 / 26 / 2005$ & 19.98 & 765.20 \\
\hline $58-35-105$ & 302801097430801 & 302801.00 & 974308.00 & 70.0 & industrial & 780.0 & 1970 & $04 / 26 / 2005$ & 15.19 & 769.99 \\
\hline 58-35-107 & 302810097430301 & 302810.00 & 974303.00 & 70.0 & industrial & 783.0 & 1969 & $04 / 26 / 2005$ & 15.79 & 769.39 \\
\hline $58-35-112$ & 302947097440301 & 302923.00 & 974348.00 & 140.0 & domestic & 817.50 & 2005 & $04 / 22 / 2005$ & 66.78 & 750.72 \\
\hline \multicolumn{11}{|l|}{ Bell County } \\
\hline $58-04-302$ & 305917097313901 & 305917.00 & 973139.00 & 148.0 & domestic & 680.0 & $12-09-1973$ & $05 / 16 / 2005$ & 98.78 & 577.89 \\
\hline 58-04-307 & 305805097310601 & 305805.00 & 973106.00 & 125.0 & domestic & 611.0 & $10-15-1970$ & 05/16/2005 & 82.45 & 531.79 \\
\hline $58-04-508$ & 305649097323501 & 305649.00 & 973235.00 & 97.0 & public supply & 618.0 & 03-14-1979 & $05 / 16 / 2005$ & 60.75 & 558.49 \\
\hline 58-04-604 & 305718097314401 & 305720.00 & 973146.00 & 128.0 & public supply & 602.0 & 01-01-1972 & 05/16/2005 & 37.79 & 562.59 \\
\hline 58-04-605 & 305729097320801 & 305729.00 & 973208.00 & 100.0 & domestic & 630.0 & 05-26-1979 & $05 / 20 / 2005$ & 42.30 & 587.20 \\
\hline 58-04-606 & 305728097315801 & 305728.00 & 973158.00 & 84.0 & domestic & 612.0 & 03-17-1971 & $05 / 20 / 2005$ & 42.80 & 570.68 \\
\hline $58-04-609$ & 305703097315901 & 305703.00 & 973159.00 & 74.0 & domestic & 590.0 & 09-24-1971 & $05 / 20 / 2005$ & 31.20 & 557.32 \\
\hline $58-04-623$ & 305624097321101 & 305626.00 & 973215.00 & 180.0 & public supply & 640.0 & 04-07-1992 & 03/01/2005 & 86.30 & 538.12 \\
\hline 58-04-702 & 305457097353601 & 305457.00 & 973536.00 & 95.0 & unused & 730.0 & 07-24-1980 & 03/01/2005 & 72.20 & 653.37 \\
\hline 58-04-811 & 305257097341401 & 305257.00 & 973414.00 & 250.0 & public supply & 787.25 & -- & $05 / 25 / 2005$ & 160.00 & 627.25 \\
\hline $58-04-812$ & 305242097340701 & 305242.00 & 973407.00 & 231.0 & public supply & 811.09 & -- & $05 / 25 / 2005$ & 200.00 & 611.09 \\
\hline $58-04-813$ & 305307097344001 & 305307.00 & 973440.00 & -- & irrigation & 773.18 & -- & $05 / 31 / 2005$ & 148.00 & 625.18 \\
\hline $58-04-814$ & 305324097342601 & 305324.00 & 973426.00 & -- & irrigation & 754.50 & -- & $05 / 31 / 2005$ & 127.88 & 626.62 \\
\hline 58-04-815 & 305406097335701 & 305406.00 & 973357.00 & -- & irrigation & 722.46 & -- & $05 / 31 / 2005$ & 99.49 & 622.97 \\
\hline $58-05-102$ & 305913097295801 & 305913.00 & 972958.00 & 152.0 & domestic & 612.0 & 04-23-1971 & $05 / 16 / 2005$ & 77.95 & 533.43 \\
\hline $58-05-103$ & 305850097294401 & 305850.00 & 972944.00 & 157.0 & domestic & 589.0 & $10-31-1971$ & 05/16/2005 & 98.90 & 490.85 \\
\hline 58-05-204 & 305956097265601 & 305956.00 & 972656.00 & 222.0 & unused & 510.0 & 06-03-1972 & 03/03/2005 & 60.00 & 455.08 \\
\hline $58-11-303$ & 305221097393201 & 305221.00 & 973932.00 & -- & domestic & 887.66 & -- & $05 / 31 / 2005$ & 73.57 & 814.09 \\
\hline $58-11-908$ & 304714097374301 & 304714.00 & 973743.00 & 293.0 & public supply & 930.26 & -- & $05 / 25 / 2005$ & 296.00 & 634.26 \\
\hline 58-11-909 & 304725097373101 & 304725.00 & 973731.00 & 450.0 & public supply & 934.39 & -- & $05 / 25 / 2005$ & 320.00 & 614.39 \\
\hline $58-12-407$ & 304931097363701 & 304931.00 & 973637.00 & 357.0 & public supply & 902.05 & -- & $05 / 25 / 2005$ & 265.00 & 637.05 \\
\hline $58-12-410$ & 304933097364501 & 304933.00 & 973645.00 & 350.0 & public supply & 890.69 & -- & $05 / 25 / 2005$ & 260.00 & 630.69 \\
\hline
\end{tabular}


Prepared by the USGS Texas Water Science Center: 8027 Exchange Drive

Austin, TX 78754-4733

Information regarding water resources in Texas is available at http: //tx.usgs.gov/ 